\title{
Extraventricular Drainage as First-Tier Management of Raised Intracranial Pressure After Severe Pediatric Trauma Brain Injury
}

\author{
Fahrudin Alić, Aldin Jusić, Hakija Bečulić
}

Department of Neurosurgery, Cantonal Hospital Zenica, Bosnia and Herzegovina
Received: December 9, 2018

Accepted: January10, 2019

Key Words: Raised Intracranial Pressure • Internal Hydrocephalus • Extraventricular Drainage.
Objective - To present the most efficatious management of raised intracranial pressure after severe trauma brain injury in a pediatric patient. Case report - A 5-year-old Caucasian presented to the emergency room due to head injury caused by falling down stairs. At the time of admission the patient was comatose with pediatric Glasgow Coma Score 6 (V-1, E-1, M-4) and signs of recent posterior head trauma. Computed tomography (CT) scan showed a multifragmented fracture of the occipital bone above the "sinus lake" causing an epidural mass, contusion in the right cerebellar hemisphere, a skull base fracture without signs of midline shifting. Six hours later, during monitoring in the intensive care unit, the patient became cardiorespiratory unstable with signs of uncal brain herniation and Cushing's triad. Control CT revealed previously verified posttraumatic changes with new signs of decompensating internal hydrocephalus. Considering that evacuation of the epidural collection and decompression of impaired bone fragments in the area of the "sinus lake" would be too risky, extraventricular drainage as the first tier management was properly selected as the option of treatment. Taking into account the hazard / benefit ratio it was shown to be the most effective form of treatment, which was confirmed after six-month follow-up without any form of neurocognitive impairment. Conclusion - Treatment of traumatic brain injury (TBI) in the pediatric population requires a multidisciplinary approach. Different mechanisms of pediatric TBI injury vary widely by age groups and its impact on the development of primary and secondary brain lesions differs. Although many general principles of managing pediatric TBI are similar to adults, cerebral plasticity is one of the features which ultimately always leaves space for a better final outcome of treatment.

\section{Introduction}

Traumatic brain injury (TBI) is the leading cause of death and disability in children. TBI in children results in a range of traumatic injuries to the scalp, skull and brain that are comparable to those in adults, but differ in both pathophysiology and management (1). The overall mortality for all pediatric head injuries requiring hospitalization has been reported at between $10 \%-13 \%$, whereas the mortality associated with severe pediatric head injury presenting with decerebrate posturing has been reported as high as $71 \%$ (2). Regarding gender, more boys were found to undergo emergency consultation and hospitalization than girls after head trauma (3). The pooled annual incidence of traumatic 
brain injury for the pediatric population ( $<15$ years of age) is $110: 100.000$ (4).

This paper aimed to present the most effective type of management of raised intracranial pressure after severe pediatric trauma brain injury.

\section{Case Report}

A 5-year-old Caucasian presented to the Emergency Room due to head injury caused by falling down stairs. At the time of admission the patient was comatose with pediatric Glasgow Coma Score (pGCS) 6 (V-1, E-1, $\mathrm{M}-4)$, isocoric and narrow pupils, afebrile, hemodynamically unstable with signs of recent posterior head trauma. Laboratory baseline findings had reference values (CellDyn Ruby, IL 60064, USA, 2013). A routine computed tomography (CT) (Siemens Somatom Definition AS 1021-012-210 746) scan showed a multifragmented fracture of the occipital bone at the level of the "sinus lake" with impaired bone fragments towards the cerebellar parenchyma which caused an epidural mass, AP diameter $10 \mathrm{~mm}$, contusion in the right cerebellar hemisphere, skull base fracture with consequent otorrhagia from the right external ear, without signs of shifting of the medio sagittal structures, and empty basal cisterns (Fig. 1).

When the patient was cleared for intubation and sedation he was admitted to the intensive care unit (ICU) for continuous monitoring of vital parameters. Six hours later the patient became cardiorespiratory unstable with signs of uncal brain herniation and Cushing's triad. Control CT confirmed the previously verified posttraumatic changes with new signs of decompensating internal hydrocephalus (Fig. 2).

Considering the evident signs of decompensating internal hydrocephalus, blood filling the upper $4^{\text {th }}$ ventricle and the extreme risk of attempting evacuation of the epidural collection and decompression of impaired

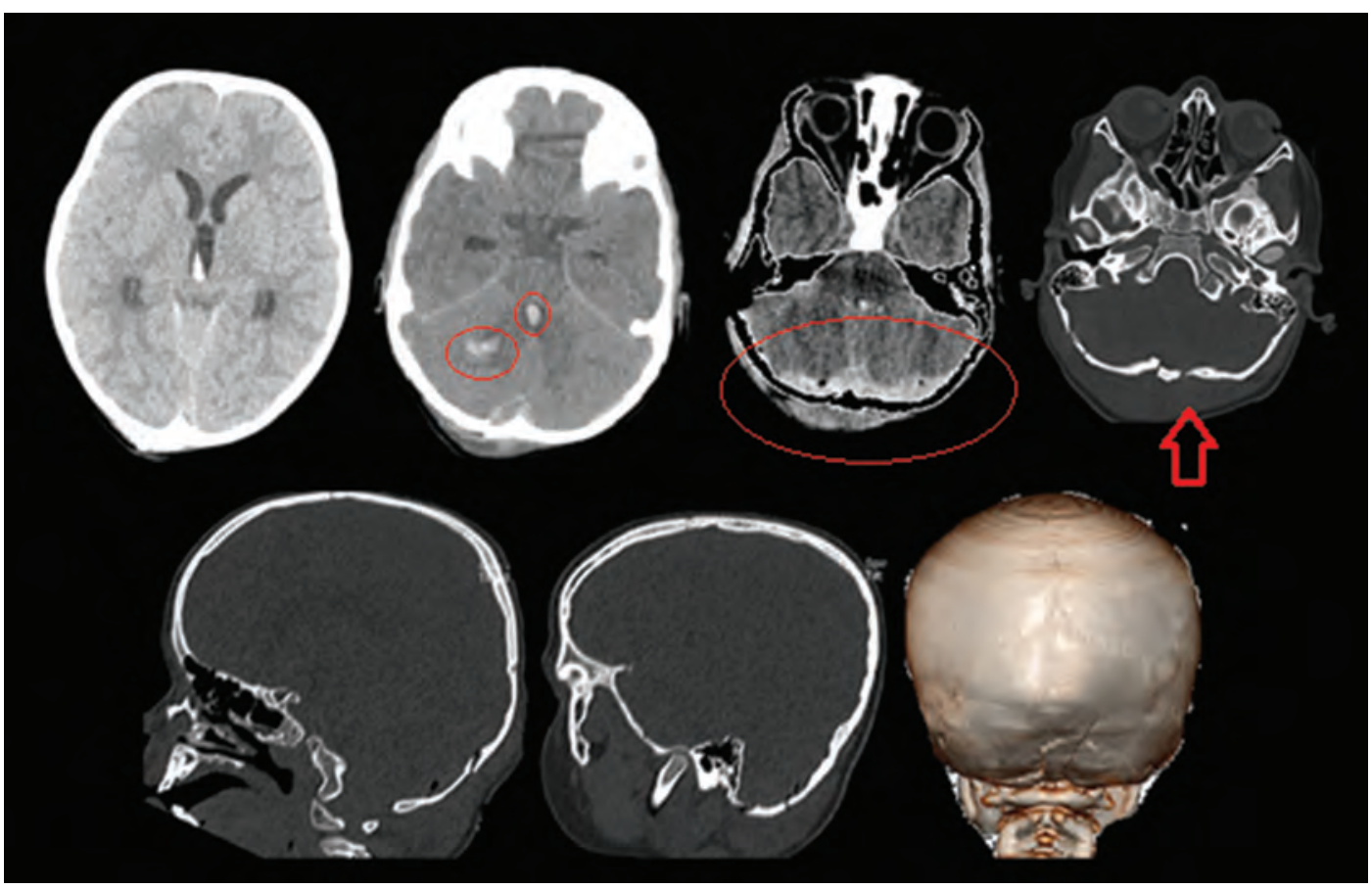

Fig. 1. Axial, Sagittal and Coronal Scan of Computed Tomography which Shows Posttraumatic Sequelae at the Level of Posterior Cranial Fossa (Department of Neurosurgery, Cantonal Hospital Zenica, 2018). 


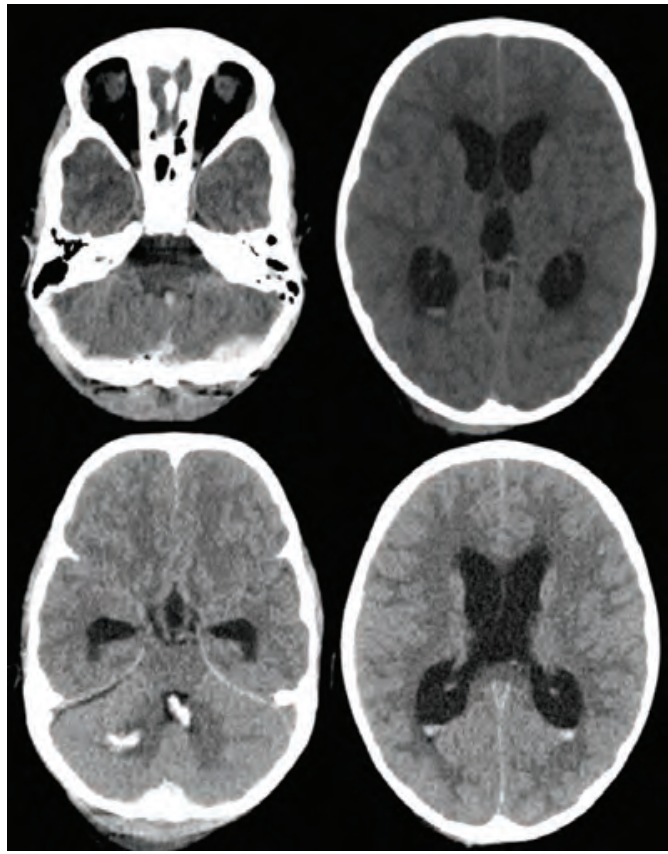

Fig. 2. Axial Computed Tomography Scan which Shows Signs of Enlargement of the Third ventricle and Temporal Horns Without the Progression of Hemorrhagic Collections in Area of Posterior Cranial Fossa (Department of Neurosurgery, Cantonal Hospital Zenica, 2018).

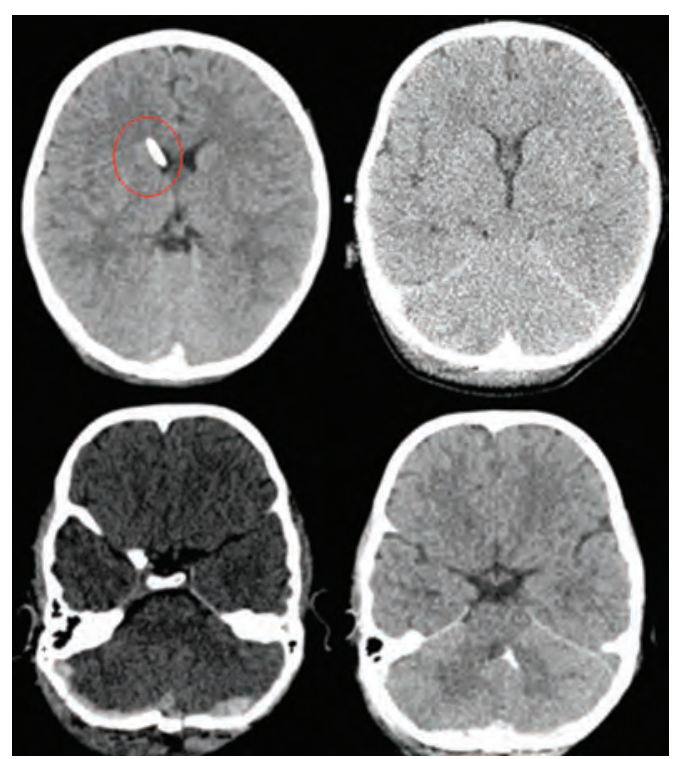

Fig. 3. Axial Computed Tomography Scan which Shows Adequately Positioned Ventricular Catheter and Unloading of Ventricular System (Department of Neurosurgery, Cantonal Hospital Zenica, 2018). bone fragments, extraventricular drainage (EVD) as the first tier management was properly selected as the most effective treatment option (Fig. 3).

After a few days in a barbiturate coma, the patient gradually woke up and the EVD was closed. Control CT was performed and revealed the adequately positioned ventricular catheter, the satisfactory position, volume and flow of the ventricular system without progression of the previously verified hemorrhagic collections in the posterior fossa cavity. Accordingly, the EVD was removed. One month after continuous parenteral antibiotic therapy and correction of electrolyte imbalance the patient's condition gradually improved. He was involved in active physical treatment, verticalized and mobilized with genuine symptoms of akinetic mutism. Magnetic Resonance imaging (MRI) (Siemens MagnetomAvanto 1,5 T, Erlangen, Germany) confirmed the correct position of the ventricular system, hemorrhagic collections of the posterior fossa cavity and deep basal structures in the resolution phase with complete thrombosis of the right sigmoid sinus, jugular bulb and consequently the welldeveloped contralateral venous collateral network (Fig. 4).

Given the surprisingly good clinical improvement and satisfactory MRI finding, the patient was discharged after two months of hospitalization with recommendations for continuation of further physical treatment and treatment by a pediatric speech therapist. After a six-month follow-up the patient was in a state of full consciousness, neurologically intact with motor strength up to normal and complete regression of signs of cerebellar syndrome. 


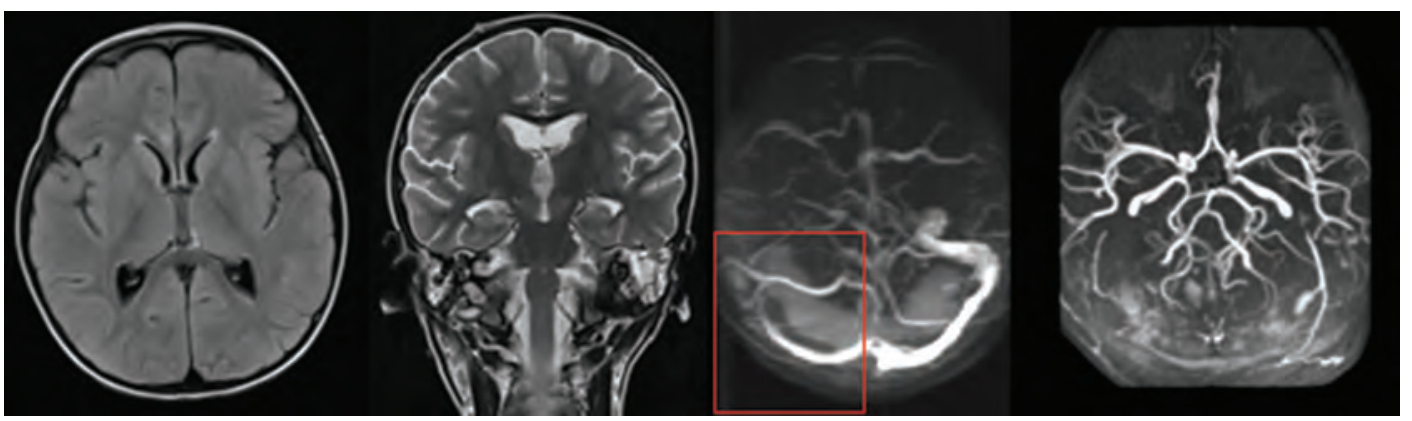

Fig. 4. Axial Magnetic Resonance Imaging. T1-Weighted Image and Coronal T2- Weighted Image Showing Correct Status of Ventricular System; Magnetic Resonance Angiography Showing Complete Thrombosis of The Right Sigmoid Sinus, Jugular Bulb and the Consequent Development of the Contralateral Venous Collateral Network (Department of Neurosurgery, Cantonal Hospital Zenica, 2018).

\section{Discussion}

Traumatic brain injury is a heterogeneous condition characterized by marked variability in etiology and treatment. Since there is a small number of studies which refer to the treatment of head injuries in the pediatric population it is important to emphasize that the still-maturing central nervous system (CNS) responds differently to injury than in adults, and that the best course of action to improve outcomes in this population is imperative (5). Although most pediatric head injuries are mild and involve only evaluation or brief hospital stays, CNS injuries are the most common cause of pediatric traumatic death (6). The death rate is higher for children younger than 4 years of age than for those 5-14 years of age (7), which confirms our case. The most common mechanisms of pediatric TBI vary according to age, and unintentional falls are the leading cause of TBI in children younger than 14 years of age (8). Boys and adolescents present more frequently to the extraventircular drainage for TBI, with a male to female ratio of 3:2 (9). As race also appears to have an impact on the risk of suffering from TBI it was concluded that African-American children have a less favorable clinical and functional outcome after TBI (10). Pediatric TBI can be categorized into two types of injury: primary - which is the consequence of the initial trauma or impact of force (epidural hematoma, subdural hematoma, subarachnoid hemorrhage, intraventricular hemorrhage, diffuse axonal injury, cortical contusion, intracerebral hematoma), and secondary - which occurs as a complication of secondary insults following primary injury(diffuse cerebral swelling, brain herniation, infarction, infection, hydrocephalus, encephalomalacia, cerebrospinal fluid leakage, leptomeningeal cyst) (11). For initial diagnostic evaluation the pGCS is commonly used to assess consciousness and to define the severity of head injury. The diagnosis of pediatric TBI is primarily made by CT of the brain and is associated with increased ICP (12). Approximately $11 \%$ of pediatric depressed cranial fractures are accompanied by dural injuries that result in intracranial lesions (13), which is why such children should be admitted to the intensive care unit (ICU) for possible progression of epidural hematoma and posttraumatic venous sinus thrombosis, as in our scenario. Posterior fossa acute epidural hematoma (AEDH) is commonly associated with an occipital fracture overlying the region from the transverse to the sigmoid sinus. It has been demonstrated that AEDH generally does not exceed the suture line, except in cases when it is of venous etiology, 
when it may extend beyond and even to the opposite side of the suture (14). According to Pediatric Guidelines from 2012 cerebrospinal fluid (CSF) drainage through an external ventricular drain in the management of high ICP in children with severe TBI, and also a lumbar drain in the case of refractory intracranial hypertension with a functioning EVD, open basal cisterns, and no signs of shifting on imaging, is fully recommended (15). Children with TBI suffer from neurocognitive impairments and are at risk for derailed academic and social development (16), which cannot be claimed for our boy.

\section{Conclusion}

Treatment of referred traumatic brain injury (TBI) in the pediatric population requires a multidisciplinary approach. Different mechanisms of pediatric TBI injury vary widely by age group and their impact on the development of primary and secondary brain lesions differs. Although many general principles of managing pediatric TBI are similar to adults, cerebral plasticity is one of the features which ultimately always leaves space for a better final outcome of treatment.

Authors' Contributions: Conception and design: FA and AJ; Acquisition, analysis and interpretation of data: FA, AJ, and HB; Drafting the article: FA; Revising the article critically for intellectual content: FA, AJ, and HB; Approved final version of the manuscript: FA and AJ.

Conflict of Interest: The authors declare that they have no conflict of interest.

\section{Reference}

1. Araki T, Yokota H, Morita A. Pediatric Traumatic Brain Injury: Characteristic Features, Diagnosis, and Management. Neurol Med Chir. 2017;57(2):82-93.

2. Bullock MR, Povlishock JT. Guidelines for the management of severe traumatic brain injury. Editor's Commentary. J Neurotrauma. 2007;24 (Sup- pl 1:2 p preceding S1). Erratum in: J Neurotrauma. 2008;25(3):276-8.

3. Langlois AJ, Rutland-Brown W, Thomas EK. Traumatic brain injury in the United States: emergency department visits, hospitalizations, and deaths. Atlanta, Ga: Dept. of Health and Human Services, Centers for Disease Control and Prevention, Division of Acute Care, Rehabilitation Research and Disability Prevention, National Center for Injury Prevention and Control; 2004.

4. Nguyen R, Fiest KM, McChesney J, Kwon CS, Jette N, Frolkis $A D$, et al. The International Incidence of Traumatic Brain Injury: A Systematic Review and Meta-Analysis. Can J Neurol Sci. 2016;43(6):774-85.

5. McCauley SR, Wilde EA, Anderson VA, BedellG, Beers SR, Campbell TF, et al. Recommendations for the use of common outcome measures in pediatric traumatic brain injury research. J Neurotrauma. 2012;29:678-705.

6. Ward JD. Pediatric Head Injury. In: Neurotrauma. Narayan RK, Wilberger JE, Povlishock JT, editors. New York: McGraw-Hill;1996. p. 859-867.

7. Keenan HT, Runyan DK, Marshall SW, Nocera MA, Merten DF, Sinal SH. A population-based study of inflicted traumatic brain injury in young children. JAMA 2003;290:621-6.

8. Injury Prevention and Control: Traumatic Brain Injury, Centers for Disease Control and Prevention [access 2018 March 12; cited 2018 November 9]. Available from: http:// www.cdc.gov/traumaticbraininjury/data/dist hosp.html.

9. Langlois JA, Rutland-Brown W, Thomas KE. The incidence of traumatic brain injury among children in the United States: Differences by race. J Head Trauma Rehabil. 2005;20:229-38.

10. Haider AH, Efron DT, Haut ER, DiRusso SM, Sullivan T, Cornwell EE $3^{\text {rd }}$. Black children experience worse clinical and functional outcomes after traumatic brain injury: an analysis of the National Pediatric Trauma Registry. J Trauma. 2007;62(5):1259-62.

11. Le TH, Gean AD. Neuroimaging of traumatic brain injury. Mt Sinai J Med. 2009;76:145-62.

12. Kannan N, Ramaiah R, Vavilala MS. Pediatric neurotrauma. Int J Crit Illn Inj Sci. 2014;4(2):131-7.

13. Vitali AM, Steinbok P. Depressed skull fracture and epidural hematoma from head fixation with pins for craniotomy in children. Childs Nerv Syst. 2008;24(8):917-23. 
14. Huisman TA, Tschirch FT. Epidural hematoma in children: do cranial sutures act as a barrier? J Neuroradiol. 2009;36(2):93-7.

15. Kochanek PM, Carney N, Adelson PD, Ashwal S, Bell MJ, Bratton S, et al; American Academy of Pediatrics-Section on Neurological Surgery; American Association of Neurological Surgeons/Congress of Neurological Surgeons; Child Neurology Society; European Society of Pediatric and Neonatal Intensive Care; Neurocritical Care Society; Pediatric Neurocritical Care Research Group; Society of Critical Care Medicine; Paediatric Intensive
Care Society UK; Society for Neuroscience in Anesthesiology and Critical Care; World Federation of Pediatric Intensive and Critical Care Societies. Guidelines for the acute medical management of severe traumatic brain injury in infants, children, and adolescents--second edition. Pediatr Crit Care Med. 2012;13(Suppl 1):S1-82.

16. Babikian T, Asarnow R. Neurocognitive outcomes and recovery after pediatric TBI: metaanalytic review of the literature. Neuropsychology. 2009;23(3):283-96. 